\title{
ON THE LEVEL SETS OF A DISTANCE FUNCTION IN A MINKOWSKI SPACE
}

\author{
RONALD GARIEPY AND W. D. PEPE
}

\begin{abstract}
Given a closed subset of an $n$-dimensional Minkowski space with a strictly convex or differentiable norm, then, for almost every $r>0$, the $r$-level set (points whose distance from the closed set is $r$ ) contains an open subset which is an $n-1$ dimensional Lipschitz manifold and whose complement relative to the level set has $n-1$ dimensional Hausdorff measure zero. In case $n=2$ and the norm is twice differentiable with bounded second derivative, almost every level set is a 1 manifold.
\end{abstract}

1. Introduction. Suppose $A$ is a closed subset of $R^{n}, n \geqq 2$, and for $x \in R^{n} \backslash A$, let $\delta(x)$ denote the distance from $x$ to $A$ in the usual metric. H. Federer [F] has shown that, for almost every $r>0, \delta^{-1}(r)$ is an Hausdorff $n-1$ rectifiable subset of $R^{n}$.

In case $n=2$, Kufarev and Nikulina [K] have shown that, for $A$ the complement of a bounded open set, the components of almost all $\delta^{-1}(r)$ are either 1 manifolds or points.

In this paper we consider the case where $A$ is a closed subset of a Minkowski space $(V,\|\cdot\|)$. We show that, if the norm is strictly convex or is differentiable, then, for almost every $r>0, \delta^{-1}(r)$ has an open subset which is a Lipschitz $n-1$ manifold and whose complement relative to $\delta^{-1}(r)$ has $n-1$ Hausdorff measure zero.

In case $n=2$ and $\|\cdot\|$ is twice differentiable with the norm of its second differential bounded on $\{y:\|y\|=1\}$, we show that $\delta^{-1}(r)$ is a 1 manifold for almost every $r>0$.

It has been called to our attention that in case $(V,\|\cdot\|)$ is $R^{2}$ with the usual norm this latter result has been announced [Fe] by S. Ferry. In fact, Ferry has communicated to us that he has proved this result for $R^{3}$ with the usual norm and has a counterexample for $R^{4}$. These results will appear in his thesis at the University of Michigan.

We would like to thank V. Klee for suggesting that we generalize our results to Minkowski spaces.

Received by the editors March 26, 1971.

AMS 1969 subject classifications. Primary 5478.

Key words and phrases. Distance function, level set, Hausdorff measure, Lipschitz manifold. 
2. Suppose $\|\cdot\|$ is a norm on $R^{n}$ and let $\phi(x)=\|x\|$ for $x \in R^{n}$. For $x, \alpha \in R^{n}$ and $t>0$, the ratio $(\phi(x+t \alpha)-\phi(x)) / t$ does not increase as $t$ decreases and hence

$$
\phi^{\prime}(x, \alpha)=\lim _{t \rightarrow 0^{+}} \frac{\phi(x+t \alpha)-\phi(x)}{t}
$$

exists for $x, \alpha \in R^{n}$. According to [R, p. 213], $\phi^{\prime}(x, \alpha)$ has the following properties:

1. $\phi^{\prime}(x, \alpha) \geqq-\phi^{\prime}(x,-\alpha)$ and

2. $\phi^{\prime}(x, \alpha)$ is an upper semicontinuous function of $x$.

Suppose $A$ is a closed subset of $R^{n}$ and, for $x \in R^{n} \mid A$, let $\delta(x)$ denote the distance from $x$ to $A$ in the metric induced by $\|\cdot\|$. Then $\delta$ is Lipschitzian with constant 1 and hence is differentiable almost everywhere in $R^{n}$.

For $x \in R^{n} \backslash A$, let $N(x)=\{y: x+\delta(x) y \in A$ and $\|y\|=1\}$ and, for $\epsilon>0$, let $N(x, \epsilon)=\left\{y:\|y\| \geqq 1\right.$ and $\left\|y-y^{\prime}\right\|<\epsilon$ for some $\left.y^{\prime} \in N(x)\right\}$.

Lemma 1. If $x \in R^{n} \mid A$ and $\alpha \in R^{n}$, then

$$
\begin{aligned}
\liminf _{\epsilon \rightarrow 0^{+}}\left\{\phi^{\prime}(y,-\alpha): y \in N(x, \epsilon)\right\} \leqq \liminf _{t \rightarrow 0^{+}} \frac{\delta(x+t \alpha)-\delta(x)}{t} \\
\leqq \limsup _{t \rightarrow 0^{+}} \frac{\delta(x+t \alpha)-\delta(x)}{t} \leqq \inf \left\{\phi^{\prime}(y,-\alpha): y \in N(x)\right\} .
\end{aligned}
$$

Proof. If $y \in N(x)$, then $x+\delta(x) y \in A$ and for any $t$,

Thus

$$
\delta(x+t \alpha) \leqq\|(x+\delta(x) y)-(x+t \alpha)\|=\|\delta(x) y-t \alpha\| .
$$

$$
\limsup _{t \rightarrow 0^{+}} \frac{\delta(x+t \alpha)-\delta(x)}{t} \leqq \phi^{\prime}(\delta(x) y,-\alpha)=\phi^{\prime}(y,-\alpha) .
$$

Suppose $\epsilon>0$. Since $A$ is closed and $\delta$ is continuous, there is an $\eta>0$ such that, for $\left\|x-x_{1}\right\|<\eta$, we have $\left\{x_{1}+\delta\left(x_{1}\right) y: y \in N\left(x_{1}\right)\right\} \subset$ $\{x+\delta(x) y: y \in N(x, \epsilon)\}$. Thus, for each $t$ such that $0<t<\eta$, there is a $y \in N(x, \epsilon)$ such that

$$
\delta(x+t \alpha)=\|(x+\delta(x) y)-(x+t \alpha)\|=\|\delta(x) y-t \alpha\|,
$$

and since $\|y\| \geqq 1$,

Hence,

$$
\frac{\delta(x+t \alpha)-\delta(x)}{t} \geqq \frac{\|\delta(x) y-t \alpha\|-\|\delta(x) y\|}{t} \geqq \phi^{\prime}(y,-\alpha) .
$$

$$
\liminf _{t \rightarrow 0^{+}} \frac{\delta(x+t \alpha)-\delta(x)}{t} \geqq \inf \left\{\phi^{\prime}(y,-\alpha): y \in N(x, \epsilon)\right\}
$$

and the lemma follows. 
Lemma 2. For $x \in R^{n} \backslash A$ and $\alpha \in R^{n}$, let

$$
g(x, \alpha)=\liminf _{\epsilon \rightarrow 0^{+}}\left\{\phi^{\prime}(y,-\alpha): y \in N(x, \epsilon)\right\} .
$$

Then $g(x, \alpha)$ is a lower semicontinuous function of $x$.

Proof. Suppose $\epsilon>0$. There is an $\eta>0$ such that $N\left(x_{1}, \epsilon / 2\right) \subset$ $N(x, \epsilon)$ whenever $\left\|x-x_{1}\right\|<\eta$. Thus

$$
\inf \left\{\phi^{\prime}(y,-\alpha): y \in N(x, \epsilon)\right\} \leqq \inf \left\{\phi^{\prime}(y,-\alpha): y \in N\left(x_{1}, \frac{\epsilon}{2}\right)\right\} \leqq g\left(x_{1}, \alpha\right)
$$

whenever $\left\|x_{1}-x\right\|<\eta$ and the lemma follows.

For $0 \neq \alpha \in R^{n}$ let $U(\alpha)=\{x: g(x, \alpha)>0\} \cap\left(R^{n} \backslash A\right)$. Then, by Lemma 2, $U(\alpha)$ is open and hence $U=\bigcup\left\{U(\alpha): 0 \neq \alpha \in R^{n}\right\}$ is open.

LEMMA 3. If $\delta$ is differentiable at $x$, then the convex hull of $N(x)$ is contained in $\{y:\|y\|=1\}$.

Proof. Let $T$ denote the differential of $\delta$ at $x$. Note that $\|T\| \leqq 1$. If $\|y\|=1$, then $\phi^{\prime}(y,-y)=-1$ and hence, by Lemma $1, T(y)=-1$ whenever $y \in N(x)$. then

If $\left\{y_{1}, \cdots, y_{k}\right\} \subset N(x)$ and $\alpha_{i} \geqq 0$ for $1 \leqq i \leqq k$ with $\sum_{i=1}^{k} \alpha_{i}=1$,

$$
1 \geqq\left\|\sum_{i=1}^{k} \alpha_{i} y_{i}\right\| \geqq\left|T\left(\sum_{i=1}^{k} \alpha_{i} y_{i}\right)\right|=\left|-\sum_{i=1}^{k} \alpha_{i}\right|=1 \text {. }
$$

THEOREM 1. Let $B=R^{n} \backslash(A \cup U)$.

(i) $\delta^{-1}(r) \cap U$ is a Lipschitz $n-1$ manifold for each $r>0$.

(ii) If $\{y:\|y\|=1\}$ is strictly convex or if $\phi$ is differentiable on $R^{n} \backslash\{0\}$, then, for almost every $r>0$, the closed set $\delta^{-1}(r) \cap B$ has $n-1$ dimensional Hausdorff measure zero.

Proof. (i) Suppose $\alpha_{1} \in R^{n},\left\|\alpha_{1}\right\|=1$, and $x \in U\left(\alpha_{1}\right)$. Let $\left\{\alpha_{1}, \alpha_{2}, \cdots\right.$, $\left.\alpha_{n}\right\}$ be a basis for $R^{n}$ with $\left\|\alpha_{i}\right\|=1$ for $1 \leqq i \leqq n$. Since $U\left(\alpha_{1}\right)$ is open, there is an $\eta>0$ such that $x+\sum_{i=1}^{n} s_{i} \alpha_{i} \in U\left(\alpha_{1}\right)$ whenever $\left|s_{i}\right|<\eta$ for $1 \leqq i \leqq n$.

For fixed $\left|s_{i}\right|<\eta, 2 \leqq i \leqq n$, the function

$$
h(s)=\delta\left(x+s \alpha_{1}+\sum_{i=2}^{n} s_{i} \alpha_{i}\right)
$$

is Lipschitzian for $|s|<\eta$ and $h^{\prime}(s)>0$ for almost every such $s$. Thus $h$ is an increasing function of $s$. Appealing to an implicit function theorem of 
W. H. Young [W, p. 230], we see that $\delta^{-1}(\delta(x)) \cap U$ is a Lipschitz $n-1$ manifold.

(ii) Suppose $\delta$ is differentiable at $x$. By Lemma 3, the convex hull of $N(x)$ is contained in $\{y:\|y\|=1\}$. Thus if $\{y:\|y\|=1\}$ is strictly convex, then $N(x)$ is a single point.

In case $\phi$ is differentiable, then $\phi^{\prime}(y, \alpha)$ is linear in $\alpha$. If $y_{1}, y_{2} \in N(x)$, then $\phi^{\prime}\left(y_{1}, y_{2}-y_{1}\right)=0$ and hence $\phi^{\prime}\left(y_{1}, y_{2}\right)=\phi^{\prime}\left(y_{1}, y_{1}\right)=1$. Thus, letting $y_{0}$ be any point of $N(x)$, we have $\phi^{\prime}\left(y,-y_{0}\right)=-1$ for each $y \in N(x)$.

Thus, in either case, we have $\sup \left\{\phi^{\prime}\left(y,-y_{0}\right): y \in N(x)\right\}=-1$ for fixed $y_{0} \in N(x)$. Since $\phi^{\prime}(y, \alpha)$ is upper semicontinuous in $y$, there is an $\epsilon>0$ such that $\sup \left\{\phi^{\prime}\left(y,-y_{0}\right): y \in N(x, \epsilon)\right\} \leqq-\frac{1}{2}$. Now, since

$$
\phi^{\prime}\left(y,-y_{0}\right) \geqq-\phi^{\prime}\left(y, y_{0}\right),
$$

we have

$$
-\inf \left\{\phi^{\prime}\left(y, y_{0}\right): y \in N(x, \epsilon)\right\} \leqq-\frac{1}{2} \quad \text { or } \quad g\left(x,-y_{0}\right) \geqq \frac{1}{2} .
$$

Thus, $x \in U$ and hence $\delta$ is not differentiable at any point of $B$. Hence the $n$ dimensional Lebesgue measure of $B$ is zero and, by the coarea formula [F], the $n-1$ dimensional Hausdorff measure of $\delta^{-1}(r) \cap B$ is zero for almost every $r>0$.

LEMMA 4. If $\phi$ is twice differentiable on $R^{n} \backslash\{0\}$ and the norm of its second differential is bounded on $\{y:\|y\|=1\}$, then there is a constant $K$ such that $\left|\delta^{2}\left(x_{1}\right)-\delta^{2}\left(x_{2}\right)\right|<K\left\|x_{1}-x_{2}\right\|^{2}$ whenever $x_{1}, x_{2} \in B$.

Proof. Let

$$
R_{1}=\sup \{\|d \phi(y)\|:\|y\|=1\} \quad \text { and } \quad R_{2}=\sup \left\{\left\|d^{2} \phi(y)\right\|:\|y\|=1\right\} .
$$

Let $K$ be any number greater than $2\left[R_{1}^{2}+R_{2}\right]$ and suppose that $x_{1}, x_{2} \in$ $R^{n} \backslash A$ with $\delta^{2}\left(x_{1}\right)-\delta^{2}\left(x_{2}\right) \geqq K\left\|x_{1}-x_{2}\right\|^{2}$. If $y \in N\left(x_{2}\right)$, then

Now

$$
\delta\left(x_{1}\right) \leqq\left\|\left(x_{2}+\delta\left(x_{2}\right) y\right)-x_{1}\right\| \text {. }
$$

$$
\begin{gathered}
\left\|\delta\left(x_{2}\right) y+\left(x_{2}-x_{1}\right)\right\|^{2} \leqq \\
\left\|\delta\left(x_{2}\right) y\right\|^{2}+2\left\|\delta\left(x_{2}\right) y\right\| \phi^{\prime}\left(y, x_{2}-x_{1}\right) \\
+2\left[R_{1}^{2}+R_{2}\right]\left\|x_{1}-x_{2}\right\|^{2} .
\end{gathered}
$$

Hence

or

$$
\begin{aligned}
\delta^{2}\left(x_{2}\right)+K\left\|x_{1}-x_{2}\right\|^{2} \leqq & \delta^{2}\left(x_{2}\right)+2 \delta\left(x_{2}\right) \phi^{\prime}\left(y, x_{2}-x_{1}\right) \\
& +2\left[R_{1}^{2}+R_{2}\right]\left\|x_{1}-x_{2}\right\|^{2}
\end{aligned}
$$

$$
2 \delta\left(x_{2}\right) \phi^{\prime}\left(y, x_{2}-x_{1}\right) \geqq\left[K-2\left[R_{1}^{2}+R_{2}\right]\right]\left\|x_{1}-x_{2}\right\|^{2} \text {. }
$$


Since $N\left(x_{2}\right)$ is compact and $d \phi$ is continuous,

$$
g\left(x_{2}, x_{1}-x_{2}\right)=\liminf _{\epsilon \rightarrow 0}\left\{\phi^{\prime}\left(y, x_{2}-x_{1}\right): y \in N\left(x_{2}, \epsilon\right)\right\}>0
$$

and $x_{2} \in U$.

THEOREM 2. If $n=2$ and $\phi$ satisfies the conditions of Lemma 4 , then $\delta^{-1}(r)$ is a 1 manifold for almost every $r>0$.

Proof. The set $B$ has 2 dimensional Lebesgue measure zero and from Lemma 4 it is clear that $\delta(B)$ has measure zero.

\section{REFERENCES}

F. H. Federer, Curvature measures, Trans. Amer. Math. Soc. 93 (1959), 418-491. MR 22 \#961.

Fe. S. Ferry, A Sard's theorem for distance functions, Notices Amer. Math. Soc. 17 (1970), 841. Abstract \#70T-G127.

K. B. P. Kufarev and N. G. Nikulina, Lebesgue measure of subsets of Euclidean space as the leading variation of the function defined by the distance to a closed set, Dokl. Akad. Nauk SSSR 160 (1965), 1004-1006 = Soviet Math. Dokl. 6 (1965), 232-234. MR 30 \#4900.

R. R. T. Rockafellar, Convex analysis, Princeton Univ. Press, Princeton, N.J., 1970.

W. F. W. Wilson, Jr., Implicit submanifolds, J. Math. Mech. 18 (1968/69), 229-236. MR 37 \#4826.

University OF KentuCKy, LeXINGton, Kentucky 40506 